\title{
Successful treatment of a case of acute myocardial infarction due to type $A$ aortic dissection by coronary artery stenting: A case report
}

\author{
ZHENG-GUI WANG* ${ }^{*}$,WEI ZHAO*, BO-TAO SHEN, YANG ZHENG and QUAN LIU \\ Cardiovascular Center, The First Affiliated Hospital of Jilin University, Changchun, Jilin 130021, P.R. China
}

Received September 4, 2014; Accepted May 15, 2015

DOI: 10.3892/etm.2015.2533

\begin{abstract}
Acute type A aortic dissection (AD) has been recognized as a potentially life threatening condition, which sometimes involves the ostium of the coronary artery and may lead to acute myocardial infarction (AMI). In patients with acute type A AD presenting with clinical signs of AMI, it is crucial to establish the diagnosis rapidly in order to proceed with the correct treatment. The present study reports the diagnosis of a rare case of acute type A AD with the typical presentation of acute inferior MI and cardiogenic shock, which was accidentally diagnosed during catheterization and treated by right coronary ostial occlusion stenting, allowing for further surgical interventions.
\end{abstract}

\section{Introduction}

Acute type A aortic dissection (AD) can sometimes involve the ostium of the coronary artery, leading to acute myocardial infarction (AMI). It is possible for the clinical symptoms of acute AD and AMI to be similar, which makes an accurate diagnosis of AD difficult. Furthermore, once the exact diagnosis is validated, management of the condition is controversial, as there are only a few reports in the literature and no current guidelines for treatment. The present report describes a case of acute $\mathrm{AD}$ with the typical presentation of acute inferior MI and cardiogenic shock that was diagnosed by inserting the catheter into a false lumen during coronary angioplasty and was successfully treated with right coronary ostial occlusion stenting as a bridge for surgery. To date, there are few reports that have described AD in combination with AMI treated by stenting (1-8). Among these reports, only one described a case

Correspondence to: Dr Quan Liu or Dr Yang Zheng, Cardiovascular Center, The First Affiliated Hospital of Jilin University, 71 Xinmin Street, Changchun, Jilin 130021, P.R. China

E-mail: quanliu666@163.com

E-mail: zhengyang@jlu.edu.cn

*Contributed equally

Key words: aortic dissection, acute myocardial infarction, stenting of acute AD complicated by AMI that was similarly diagnosed by accidentally inserting the catheter into a false lumen during catheterization (9).

\section{Case report}

A 51-year-old man with a history of hypertension was transferred to the Emergency Department following the sudden onset severe chest pain, which lasted $7 \mathrm{~h}$. The patient had been diagnosed with AMI and accepted thrombolytic therapy with urokinase in the local hospital and was then transferred to the First Affiliated Hospital of Jilin University (Changchun, China) in order to undergo a primary percutaneous coronary intervention (PCI). The blood pressure of the patient was at $86 / 60 \mathrm{mmHg}$ with continuous dopamine administration (5 $\mu \mathrm{g} / \mathrm{kg} / \mathrm{min}$ ) and his heart rate was $82 \mathrm{bpm}$. Other physical examination findings appeared normal, with the pulse within the normal range and no aortic regurgitation; however, the electrocardiograms (ECGs) showed a marked ST-segment elevation in leads II, III, aVF, $\mathrm{V}_{3 \mathrm{R}}-\mathrm{V}_{5 \mathrm{R}}$ and a reciprocal ST-segment depression in lead $\mathrm{V}_{4}-\mathrm{V}_{6}$ (Fig. 1). The levels of cardiac troponin I and D-dimers were also elevated. The personnel of the Emergency Department considered the diagnosis of AMI and therefore referred the patient directly to the catheterization laboratory.

During primary coronary angioplasty, the left coronary arteries were found to be normal (Fig. 2A), while the right coronary artery (RCA) was completely occluded (as found later, the catheter was in the wrong lumen) (Fig. 2B). Stent implantation was subsequently attempted by placing a guiding catheter into the true lumen (Fig. 2C), which, instead of showing RCA stenosis, revealed dissection over the ostium of the RCA, indicating the diagnosis of $\mathrm{AD}$. In order to promote rapid recovery from the cardiogenic shock, primary angioplasty was performed using the implantation of three drug-eluting stents in the RCA that reached the ostium. Following treatment by direct stenting, the anterograde coronary blood flow was completely restored and myocardial perfusion improved (Fig. 2D). Following the procedure, the cardiac hemodynamics of the patient were also stabilized. As a result, the dose of dopamine was gradually reduced, as the patient had a blood pressure of $110 / 70 \mathrm{mmHg}$ while on continuous dopamine at a dose of $3 \mu \mathrm{g} / \mathrm{kg} / \mathrm{min}$. Aortic computed tomography angiography revealed Stanford type A AD (Fig. 3A and B). The 


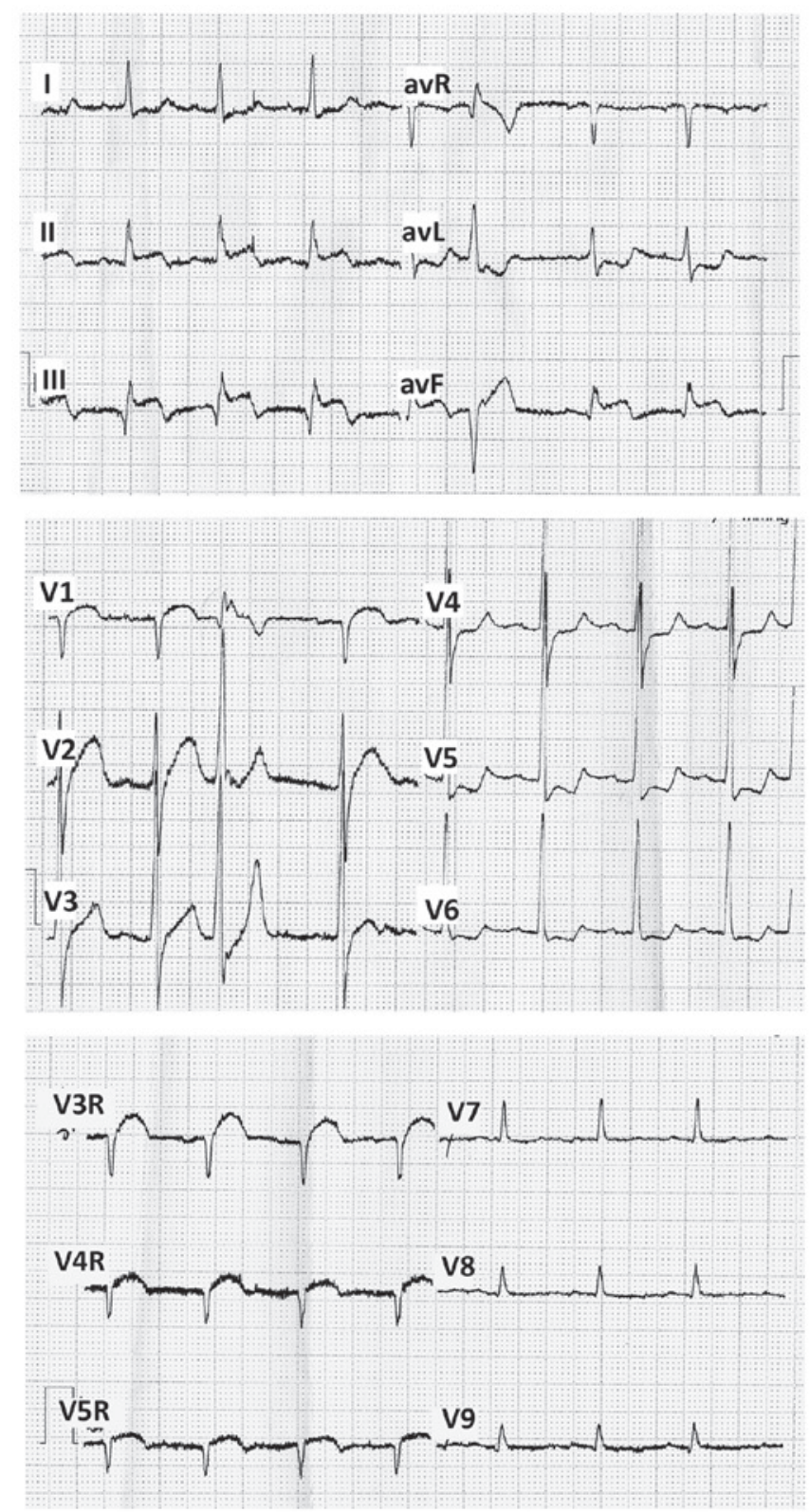

Figure 1. Electrocardiographic examination of patient on admission showed marked ST-segment elevation in leads II, III, aVF and $\mathrm{V}_{3 \mathrm{R}}-\mathrm{V}_{5 \mathrm{R}}$ and a reciprocal ST-segment depression in leads $\mathrm{V}_{4}-\mathrm{V}_{6}$. Occasional premature beats could also be observed.

patient was then immediately transferred to the Department of Cardiac Surgery, which confirmed the diagnosis of dissection of the ascending aorta with involvement of the RCA. A tetrafurcated graft with stented elephant trunk implantation (Sun's procedure) was used, followed by surgical replacement of the partial ascending aorta and aortic arch under stable hemodynamic conditions. Following surgery, the patient was stabilized and shortly admitted to the Intensive Care Unit.

\section{Discussion}

Complications of acute AD in the setting of AMI are relatively rare, with a reported incidence of $1-3 \%(10,11)$. The present study reported a case of acute AD combined with AMI, which was successfully treated by coronary artery stenting. The diagnosis of acute inferior and right ventricular MI was determined based on the clinical symptoms of the patient, the ST-segment elevation observed via ECG and the elevated serum levels of troponin. The guiding catheter was accidentally placed in a false lumen and the dissection was noted, since stenosis was absent in the RCA when the catheter was inserted into the true lumen and dissection over the ostium of the RCA was revealed. In this case, the possibility of iatrogenic coronary dissection was ruled out. To promote rapid recovery from the cardiogenic shock, a direct PCI was performed as a bridge to further surgical interventions. In cases such as the present one, in which the patient exhibits shock, a correct diagnosis may be even more difficult to make, and direct stenting may be required to save the patient's life during angioplasty.

In previous years, a limited number of cases of Stanford type $\mathrm{A} \mathrm{AD}$ in combination with $\mathrm{MI}$ that were treated with coronary stenting have been reported (1-8). Furthermore, only one case was diagnosed in a similar manner to the patient 

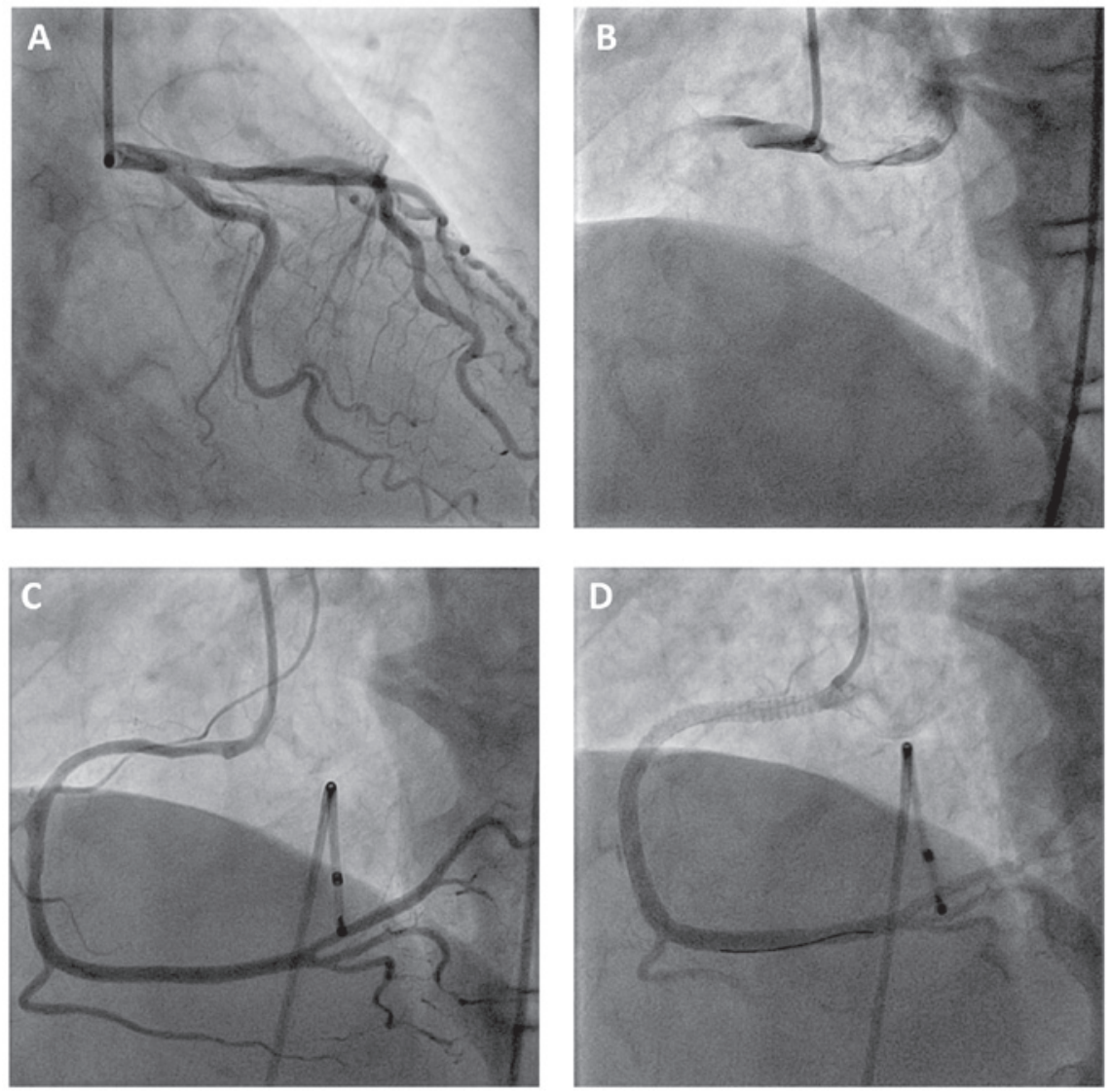

Figure 2. Coronary angiogram and percutaneous coronary intervention procedures. Coronary angiogram showed (A) the normal left coronary artery and (B) total occlusion of the RCA with the catheter inserted in the false lumen. (C) The guiding catheter was placed in the true lumen. (D) The placement of three drug-eluting stents in the RCA that also reached the ostium was sufficient to completely restore the anterograde coronary flow. RCA, right coronary artery.
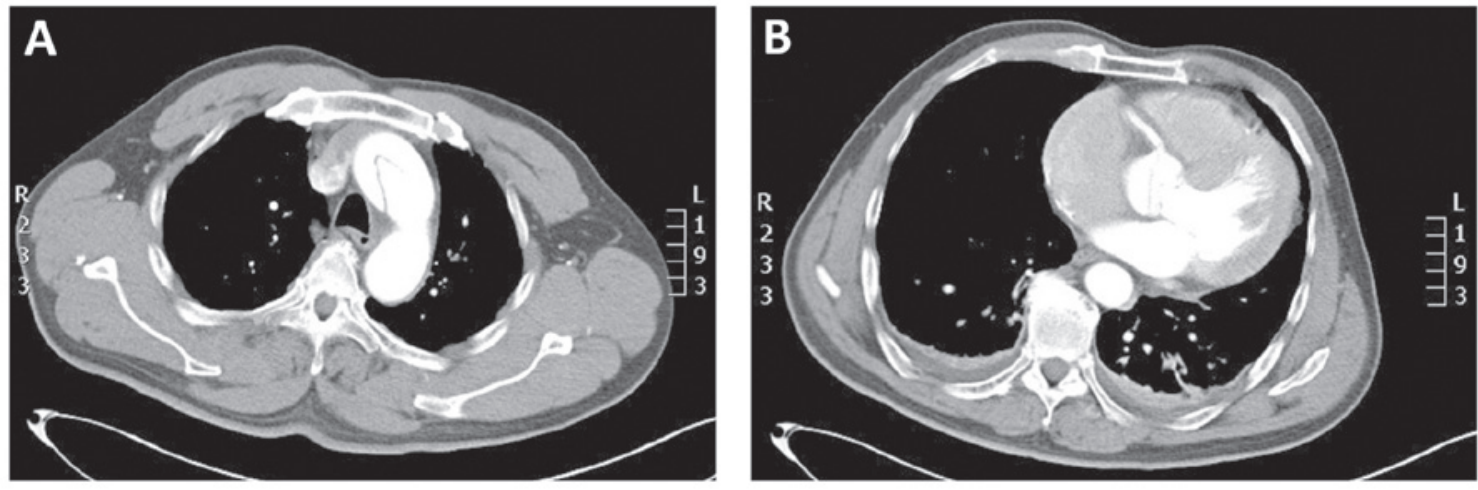

Figure 3. Computed tomography scan of the chest of the patient. (A) A flap was observed at the aortic arch. (B) Stents can be noted in the right coronary artery.

described in the present study (9). Physicians should therefore be aware of primary and secondary ischemic heart diseases that are complicated by AD. Thorough physical examinations along with transthoracic echocardiograms should be performed before catheterization to exclude Stanford type A AD with AMI. Once the correct diagnosis is validated, a treatment strategy should be determined. Surgery has been recommended as the treatment of choice for patients with typical acute type A AD (12); however in cases of hemodynamic instability due to the complication of AMI, as is in the present case, a primary PCI should be considered as a bridge approach to not only stabilize the cardiac hemodynamics of the patient but also gain some time before surgery can be performed.

\section{Acknowledgements}

The authors would like to thank Medjaden Bioscience Limited (Hong Kong SAR, China) for their assistance in the preparation of this manuscript. 


\section{References}

1. Cardozo C, Riadh R and Mazen M: Acute myocardial infarction due to left main compression aortic dissection treated by direct stenting. J Invasive Cardiol 16: 89-91, 2004.

2. Barabas M, Gosselin G, Crépeau J, Petitclerc R, Cartier R and Théroux P: Left main stenting - as a bridge to surgery - for acute type A aortic dissection and anterior myocardial infarction. Catheter Cardiovasc Interv 51: 74-77, 2000.

3. Yunoki K, Naruko T, Itoh A, et al: Stenting of right coronary ostial occlusion due to thrombosed type A aortic dissection: One-year follow-up results. J Cardiol 1: e166-e170, 2010.

4. Shimamura J, Kubota H, Tonari K, et al: Acute aortic dissection with left main coronary malperfusion treated with precedent stenting followed by a definitive surgery. Kyobu Geka 63: 537-541, 2010 (In Japanese).

5. Ohara Y, Hiasa Y and Hosokawa S: Successful treatment in a case of acute aortic dissection complicated with acute myocardial infarction due to occlusion of the left main coronary artery. J Invasive Cardiol 15: 660-662, 2003.
6. Lentini S, Specchia L, Cricco A, et al: Hybrid management of acute type A aortic dissection presenting as acute coronary syndrome. Int J Cardiol 167: e85-e87, 2013.

7. Ravandi A and Penny WF: Percutaneous intervention of an acute left main coronary occlusion due to dissection of the aortic root. JACC Cardiovasc Interv 4: 713-715, 2011.

8. Camaro C, Wouters NT, Gin MT and Bosker HA: Acute myocardial infarction with cardiogenic shock in a patient with acute aortic dissection. Am J Emerg Med 27: 899, 2009.

9. Chan KC, Wu DJ, Ueng KC, et al: Acute aortic dissection developing acute myocardial infarction diagnosed by accidentally inserting the catheters into the false lumen during catheterization: A pitfall and rare image. Jpn Heart J 44: 583-585, 2003.

10. DeSanctis RW, Doroghazi RM, Austen WG and Buckley MJ: Aortic dissection. N Engl J Med 317: 1060-1067, 1987.

11. Neri E, Toscano T, Papalia U, et al: Proximal aortic dissection with coronary malperfusion: Presentation, management, and outcome. J Thorac Cardiovasc Surg 121: 552-560, 2001.

12. Prêtre R and Von Segesser LK: Aortic dissection. Lancet 349: 1461-1464, 1997. 